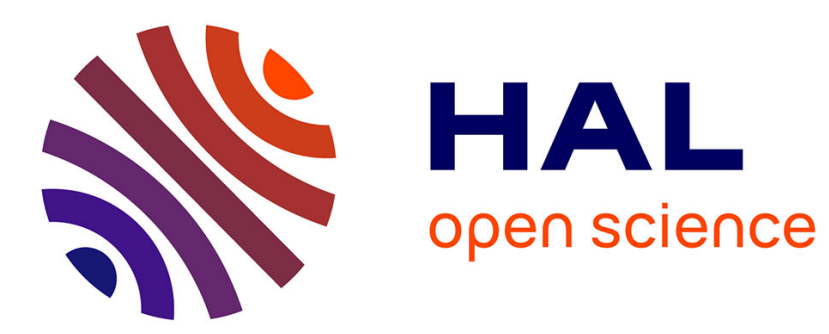

\title{
Three-dimensional curvilinear device reconstruction from two fluoroscopic views
}

Charlotte Delmas, Marie-Odile Berger, Erwan Kerrien, Cyril Riddell, Yves Trousset, René Anxionnat, Serge Bracard

\section{- To cite this version:}

Charlotte Delmas, Marie-Odile Berger, Erwan Kerrien, Cyril Riddell, Yves Trousset, et al.. Threedimensional curvilinear device reconstruction from two fluoroscopic views. SPIE, Medical Imaging 2015: Image-Guided Procedures, Robotic Interventions, and Modeling, Feb 2015, San Diego, CA, United States. pp.94150F, 10.1117/12.2081885 . hal-01139284

\section{HAL Id: hal-01139284 \\ https://inria.hal.science/hal-01139284}

Submitted on 3 Apr 2015

HAL is a multi-disciplinary open access archive for the deposit and dissemination of scientific research documents, whether they are published or not. The documents may come from teaching and research institutions in France or abroad, or from public or private research centers.
L'archive ouverte pluridisciplinaire HAL, est destinée au dépôt et à la diffusion de documents scientifiques de niveau recherche, publiés ou non, émanant des établissements d'enseignement et de recherche français ou étrangers, des laboratoires publics ou privés. 


\title{
Three-dimensional curvilinear device reconstruction from two fluoroscopic views
}

\author{
Charlotte Delmas ${ }^{a b}$, Marie-Odile Berger ${ }^{b c}$, Erwan Kerrien ${ }^{b c}$, Cyril Riddell ${ }^{a}$, Yves Trousset $^{a}$, \\ René Anxionnat ${ }^{d}$, Serge Bracard ${ }^{d}$ \\ ${ }^{a}$ GE Healthcare, Buc, France; \\ ${ }^{b}$ Loria, Vandoeuvre-les-Nancy, France \\ ${ }^{c}$ Inria, Vandoeuvre-les-Nancy, France \\ ${ }^{d} \mathrm{CHU}$, Nancy, France
}

\begin{abstract}
In interventional radiology, navigating devices under the sole guidance of fluoroscopic images inside a complex architecture of tortuous and narrow vessels like the cerebral vascular tree is a difficult task. Visualizing the device in 3D could facilitate this navigation. For curvilinear devices such as guide-wires and catheters, a 3D reconstruction may be achieved using two simultaneous fluoroscopic views, as available on a biplane acquisition system. The purpose of this paper is to present a new automatic three-dimensional curve reconstruction method that has the potential to reconstruct complex 3D curves and does not require a perfect segmentation of the endovascular device. Using epipolar geometry, our algorithm translates the point correspondence problem into a segment correspondence problem. Candidate 3D curves can be formed and evaluated independently after identifying all possible combinations of compatible 3D segments. Correspondence is then inherently solved by looking in 3D space for the most coherent curve in terms of continuity and curvature. This problem can be cast into a graph problem where the most coherent curve corresponds to the shortest path of a weighted graph. We present quantitative results of curve reconstructions performed from numerically simulated projections of tortuous 3D curves extracted from cerebral vascular trees affected with brain arteriovenous malformations as well as fluoroscopic image pairs of a guide-wire from both phantom and clinical sets. Our method was able to select the correct 3D segments in $97.5 \%$ of simulated cases thus demonstrating its ability to handle complex 3D curves and can deal with imperfect $2 \mathrm{D}$ segmentation.
\end{abstract}

\section{CONTEXT}

In interventional radiology, navigating devices under the sole guidance of fluoroscopic images inside a complex architecture of tortuous and narrow vessels like the cerebral vascular tree is a difficult task. Visualizing the device in 3D could facilitate this navigation. For curvilinear devices such as guide-wires and catheters, a 3D reconstruction may be achieved using two simultaneous fluoroscopic views, as available on a biplane acquisition system. Most proposed methods for curvilinear devices reconstruction from two fluoroscopic views require a prior segmentation of the device. Using epipolar geometry $[1,2]$ solve the point correspondence problem by enforcing an ordering along a provided B-spline fit of the segmented device in the image pair. Achieving such fits can be difficult since the $2 \mathrm{D}$ projection of a $3 \mathrm{D}$ curve is not necessarily smooth and may present high curvatures going as far as forming cusps. In their work [3] use intersection of back-projection rays cast from segmented points in the image pair to reconstruct a 3D point cloud containing the 3D curve. This curve is recovered by finding a minimum spanning tree minimizing the distance between points. However distance alone is not sufficient to recover the correct curve in some ambiguous cases (e.g. loops). All these methods require a correct prior segmentation of the device which in the case of fluoroscopic images, characterized by a low signal to noise ratio, is a difficult task addressed by many authors $[4,5,6]$. The problem of stereo correspondence for curvilinear structures in a noisy environment was also thoroughly studied by the computer vision community $[7,8]$. Imposing left-to-right ordering or limiting disparities (in orientation or intensity for instance) are commonly used constraints to prevent obvious false correspondences. However the image planes are almost orthogonal during biplan neuro-interventions which is the worst configuration to use left-to-right constraints or the disparities on $2 \mathrm{D}$ orieantations. As for intensity, fluoroscopic images are too corrupted with noise to use their disparity as a matching constraint. We present in this paper a new automatic three-dimensional curve reconstruction method, 
that has the potential to reconstruct tortuous $3 \mathrm{D}$ curves without necessarily requiring a perfect segmentation of the endovascular device. Instead of basing our analysis solely on 3D points, we choose to reconstruct higher level features in the form of 3D segments [8]. This allows for the quantification of curvilinear continuity as a function of both the Euclidian distance between segment end-points and the alignment of the segments tips. Introducing alignment information has the advantage to better handle ambiguous situations and makes our method more robust to imperfect 2D segmentation. In this paper, we describe how those 3D segments are formed and how, using a graph representation, we find the best ordering and orientations for those 3D segments supporting the $3 \mathrm{D}$ curve corresponding to the device.

\section{METHODS}

As illustrated Figure 1 that two projections of the same curve do not necessarily back-project into a single 3D curve but rather into a set of curves. Superfluous curves are the result of alternative matching possibilities existing between both projected curves. Following [8] we think that matching and reconstruction are two sides of the same coin and should be addressed together. We therefore look at the problem of retrieving the curve corresponding to the device as a curve extraction task that can be solved using the smoothness properties of $3 \mathrm{D}$ curves. We also take advantage from the fact that only one curve has to be recovered, which provides us with additional constraints. In Figure 1 we see that alternative matches do not necessarily involve the entire projected curve but only portions of it. The corresponding 3D curve portions we qualify as ambiguous cannot be recovered in a straightforward manner and need to be addressed specifically. They project into the epipolar bands highlighted in red and back-project forming the red curves in the right image. The remaining portions projecting within the yellow epipolar bands back-project on the contrary into single continuous curves we thus qualify as unambiguous. We assume unambiguous curve portions necessarily result from a correct match and must consequently be part of the final solution. Therefore the search for the global 3D curve can be decomposed into separate searches for the ambiguous curve portions in-between to the unambiguous portions.
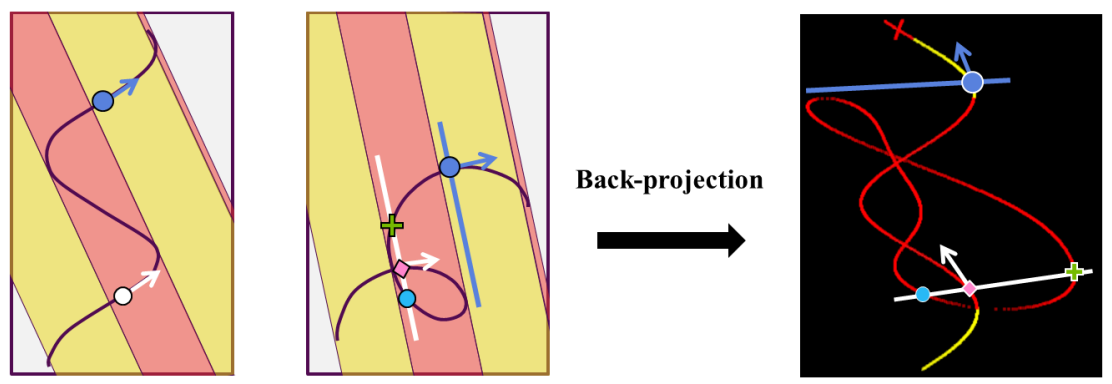

Figure 1: From left to right : 2 projective views of a 3D helical curve and the intersection of their back-projection rays. The white point in the left projection can be matched with three points lying on its associated white epipolar line in the right projection. The resulting 3D points are shown in the 3D point cloud in the right image of the figure. In this image the red $3 \mathrm{D}$ curves project in both views within the red bands while the yellow points project within the yellow bands.

\subsection{Method overview}

The method requires as input a segmentation of the device as a set of pixels (typically a skeleton) in a calibrated pair of fluoroscopic views. For simplicity segmentation was performed on rectified images where pairs of corresponding epipolar lines can be uniformly sampled as pairs of horizontal lines of pixels with the same vertical index in both images. The method starts off by a pre-structuring step, operated in both views simultaneously, that cuts the input segmentation into 2D curve segments. These 2D segments are then matched using epipolar geometry resulting in an unstructured set of 3D segments. Based on this set of 3D segments our method (i) produces for each ambiguous portion candidate 3D curves as subsets of 3D segments (ii) evaluates each candidate 3D curve (iii) selects one candidate 3D curve per ambiguous portion to recover the curve corresponding to the 
device. In step (i) a connectivity constraint between the 3D segments are applied in order to limit the number candidate curves to evaluate. In step (ii) 3D segments are ordered and oriented such that a function of both the Euclidian distance and curvature between their connected extremities is minimized. Steps (i) and (ii) are applied to each identified ambiguous portions in parallel. Step (iii) selects a single candidate curve per ambiguous portion by considering the adjacent unambiguous portions using the same minimization criteria as in step (ii).

\subsection{From 3D points to $3 \mathrm{D}$ curve segments}

A left-to-right analysis along the epipolar lines of the incoming segmentation from both views allows for a decomposition of this segmentation into $2 \mathrm{D}$ curve segments. In what follows, the term segment will denote a curve segment (in 2D or 3D). A pair of corresponding epipolar lines [9] intersects the segmentation in $m$ points in one view and $n$ points in the other view. We define $[m, n]$ its characteristic. All consecutive pairs of corresponding epipolar lines from top to bottom in the projection image that have the same characteristic $[m, n]$ can be grouped to form a pair of corresponding sets of epipolar lines with the same characteristic $[m, n]$ that we shall call band from now on. Intersection of a band with the segmentation produces $m 2 \mathrm{D}$ segments in the first view and $n$ in the second one where each 2D segment is made of points that are connected in the segmentation. If this is not the case, the band is divided into two sub-bands at the location of discontinuity. Figure 3 illustrates this concept: given a band with characteristic $[m, n]$ a total number of $m \times n$ BD segments can be formed. Three cases are considered :

i. Bands with $m=0$ or $n=0$ don't produce any 3D segments and therefore won't be further considered.

ii. Bands with $m=1$ and $n=1$ produce a single 3D segment. This 3D segment forms an unambiguous portion of the curve. Therefore we will call such bands unambiguous.

iii. All other configurations produce more than one 3D segment and are thus considered ambiguous.

Let us point out that the projection of an ambiguous 3D curve portion often span more than one ambiguous epipolar band. We shall call ambiguous group the union of all adjacent ambiguous bands spanning the projection of a single ambiguous curve portion. A 3D curve spans alternatively ambiguous and unambiguous portions portions of curves. Similarly, successive bands in the image alternate between groups of ambiguous bands, separated by singular unambiguous bands. Figure 2 provides the example of two groups $G_{1}$ and $G_{2}$ that correspond to the back-projection of two distinct ambiguous groups. Finally, we ignore bands spanning less than 3 epipolar lines because they provide inaccurate 3D curvature information. These small bands correspond in the example of Figure 2 to junctions between the different 3D segment.

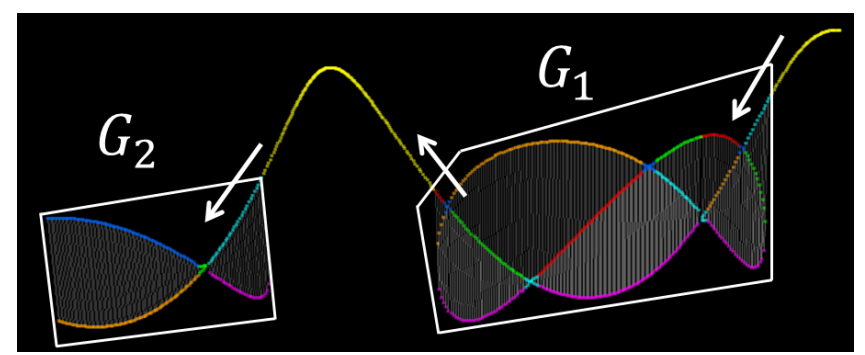

Figure 2: Reconstruction possibilities of an helical curve from two views. The two yellow curve portions are unambiguous. They act as a natural separator for the two ambiguous portions $G_{1}$ and $G_{2}$ : white arrows mark those transitions. The vertical white stripes correspond to the back-projection of epipolar lines sampled in one of the two views. They connect alternative matches for a segment of this view. 


\subsection{Graph formulation}

Within one ambiguous group, the search for candidate 3D curves can be performed efficiently by means of a graph formulation of the problem. Let us consider a graph where nodes correspond to all 3D segments produced in the group. Each candidate curve is a sequence of $3 \mathrm{D}$ segments and therefore corresponds to a path in the graph. It can be evaluated by weighting the edges of the graph with a connection quality measure that is a function of both the Euclidian distance and the curvature between two 3D segments. We consider a "good" path minimizes the sum of the weights along the path thus reflecting the continuity and smoothness of a 3D curve. However, within a complete graph, possible paths range from the shortest ones consisting of a single node to the longest one visiting every node of the graph. Let $N_{s}$ be the number of nodes. Then the total number of paths (without counting symmetric versions) adds up to $N_{\text {paths }}=\frac{1}{2} \sum_{s=1}^{N_{s}} \frac{N_{s} !}{\left(N_{s}-s\right) !}$. Allowing for any combination of nodes to form a path is both computationally very expensive and unrealistic. Indeed the expected 3D curve most probably is neither supported by every single 3D segment produced by the ambiguous group, as we saw with the example figure 1 , nor is it made only of a single 3D segment. We therefore introduce a strategy to prune edges in the graph as to extract only candidate 3D curves deemed relevant. This strategy does not depend on some threshold but is directly related to the projection of 3D curves in general.

\subsubsection{Extracting candidate 3D curves}

A 3D curve usually doesn't completely occlude itself when projected onto a plane. On the contrary, overlaps are rare and mainly appear as cusps or loop intersections in the projective image. This projective effect most often concerns a negligible portion of the 3D curve. Based on this observation, we deem relevant candidate 3D curves that minimize the number of overlaps in the two projective planes. Since we construct a 3D curve from $3 \mathrm{D}$ segments this task boils down to selecting 3D segments that do not project onto the same 2D segments in one of the two views. Within a group, 3D segments originating from different bands never overlap. This constraint is therefore to be applied between 3D segments generated by the same ambiguous epipolar band. Let us define assumption (1) stating that: No overlaps are permitted in any of the two projections. We translate the constraint in the graph by keeping only edges between nodes corresponding to 3D segments that are mutually compliant with assumption (1). A subset of 3D segments satisfying assumption (1) thus forms a complete subgraph. Therefore the curves most covering the segmentation are given by maximal cliques. We recall maximal cliques are complete sub-graphs that cannot be extended with additional nodes without losing the 'complete' property. Since assumption (1) acts at the band level, these maximal cliques can be computed efficiently within each band of the group separately and then recombine those results to get the maximal cliques of the graph that spans the whole ambiguous group. Considering assumption (1), $N_{\text {candidates }}$ (see eq(1)) candidate curves can be extracted depending on the characteristics of the considered bands. Each candidate curve is then composed of the same number of 3D segments $N_{\text {segments }}$ (see eq(2)). Examples given in Figure 3 highlight the pros and cons of assumption (1). We illustrate the case (a) where there is an actual overlap in one of the projections and another case (b) with a segmentation error. Assumption (1) in these two examples will result in either $A_{1}$, $B_{1}$ or $C_{1}$ being left unmatched for each maximal clique (see Figure d). In (a) it may be questionable to apply assumption (1) while in the (b) case it would be a good thing to have $A_{1}$ left unmatched. An indicator for the existence of an overlap is when an ambiguous band has a characteristic with $m \neq n$. In such bands we could decide to be less restrictive on the no-overlap constraint and decide to allow for $|m-n|$ overlaps in the view where there are $\min (m, n) 2 \mathrm{D}$ segments. We will refer to this relaxed version of assumption (1) with a larger combinatory as assumption (2).

$$
\begin{aligned}
N_{\text {candidates }} & =\prod_{b=1}^{B} \frac{! \max \left(m_{b}, n_{b}\right)}{!\left|m_{b}-n_{b}\right|} \\
N_{\text {segments }} & =\sum_{b=1}^{B} \min \left(m_{b}, n_{b}\right)
\end{aligned}
$$




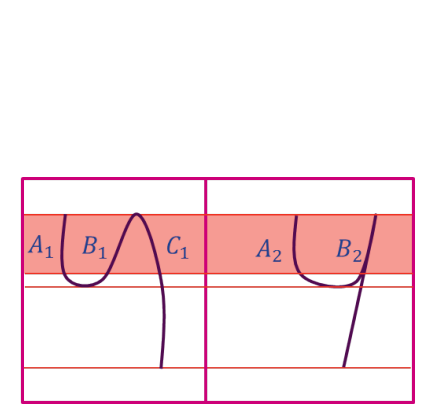

(a)

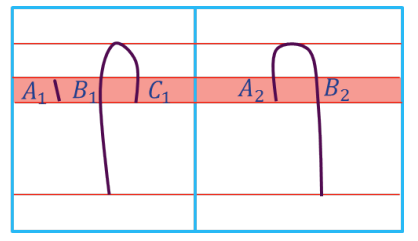

(b)

\begin{abstract}
3D segments
$S_{1}: A_{2} \leftrightarrow A_{1}$

$S_{2}: A_{2} \leftrightarrow B_{1}$

$S_{3}: A_{2} \leftrightarrow C_{1}$

$S_{4}: B_{2} \leftrightarrow B_{1}$

$S_{5}: B_{2} \leftrightarrow B_{1}$

$S_{6}: B_{2} \leftrightarrow C_{1}$
\end{abstract}

(c)

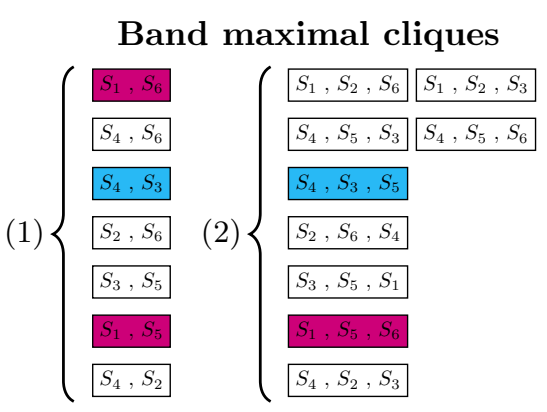

(d)

Figure 3: (a) : example of an overlap in the red epipolar band with characterstic 3, 2. (b) : example with no overlap but with $A_{1}$ a segmentation error making the characteristic of the red band also 3,2.(c): all possible matchings. (d): maximal cliques for the red band in (a) and (b) using assumption (1) or (2). Those containing the correct matches are highlighted in purple for (a) and blue for (a) . (a) + (1) : a segment is missing. (a) $+(2)$ : the segmentation error was included.

\subsubsection{Evaluating candidate $3 \mathrm{D}$ curves}

At this stage, each maximal clique is an unordered set of 3D segments. We here apply of initial hypothesis that a single curve is to be reconstructed, therefore only one single continuous curve is to be recovered from each ambiguous group. We evaluate each candidate 3D curve by finding the arrangement and orientation of its 3D segments such that it minimizes a function of both length and curvature estimated in-between the 3D segments. This tasks amounts to finding the shortest Hamiltonian path in the corresponding maximal clique. This path visits every node of the maximal clique and is the shortest in the sense that it minimizes the sum of weights along the path. In order to retrieve segments that are oriented, we produce two versions a 3D segment for each node. Each version is set with a different starting extremity. This trick enables us to explicitly represent the 4 possible connections between two 3D segments in the graph as illustrated in figure (4).
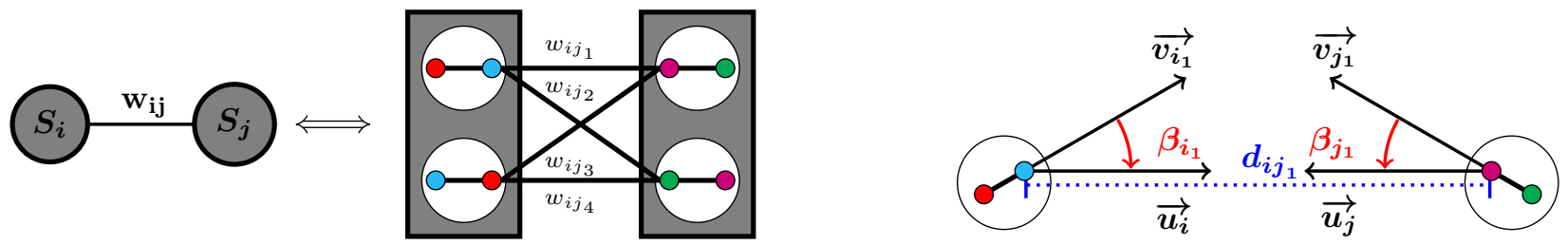

Figure 4: Two 3D segments $S_{i}$ and $S_{j}$ linked by a line segment of length $d_{i j}$ joining a pair of extremities. $\overrightarrow{v_{i}}\left(\right.$ resp. $\overrightarrow{v_{j}}$ ) is the orientation computed at the selected extremities. $\beta_{i}$ and $\beta_{j}$ are respectively the positive oriented angles $\left(\overrightarrow{v_{i}}, \overrightarrow{u_{i}}\right)$ and $\left(\overrightarrow{v_{j}}, \overrightarrow{u_{j}}\right)$ where $\overrightarrow{u_{i}}=-\overrightarrow{u_{j}}$ lie facing each other at both ends of the line segment linking the 2 extremities.

We compute the weight of an edge as a linear combination (see eq(3)) of two functions $f$ (see eq(4)) and $g$ (see eq.(5)). $f$ penalizes long distances between 2 connecting extremities while $g$ penalizes strong bending. In order to tackle the reconstruction noise, we model an extremity over a fixed length by a centroid point and an orientation vector $\vec{v}$ estimated over that same length using linear regression. Distance $d$ used in function $f$ is estimated by the Euclidian distance between the two centroid points. Coefficient $\sigma$ tunes the notion of near-far extremities. The connection between two extremities is approximated by a straight line connecting their respective centroids. The more the connection line deviates from the orientation of both extremities, the stronger the misalignment and thus the inherent bending. This deviation is characterized by the two oriented angles $\beta_{i_{k}}$ and $\beta_{j_{k}}$ as shown in figure 4. Other strategies [7] could be used for the estimation of $d$ and $\beta$ using more sophisticated approximation models such as osculating circles which take into account the bend of the curve at the point of connection. However these models are really useful when dealing with multiple branching structures as opposed to dealing with a single curve like we do which is a simpler problem. 


$$
\begin{aligned}
w_{i j_{k}}\left(d_{i j_{k}}, \beta_{i_{k}}, \beta_{j_{k}}\right) & =\alpha f\left(d_{i j_{k}}\right)+(1-\alpha) g\left(\beta_{i_{k}}, \beta_{j_{k}}\right), \alpha \in[0,1] \\
f(d) & =1-\exp \left(\frac{-d^{2}}{\sigma^{2}}\right), \sigma \in \mathbb{R} \\
g\left(\beta_{1}, \beta_{2}\right) & =\frac{1}{2}\left(1-\cos \left(\max \left(\beta_{1}, \beta_{2}\right)\right)\right)
\end{aligned}
$$

\subsection{Recovering the 3D curve corresponding to the device}

We now have a set of evaluated candidate curves per ambiguous group. We further evaluate each ambiguous candidate curve portion locally by extending it on both sides with the adjacent unambiguous portions. These were reconstructed from adjacent unambiguous bands in the image. The cost of each candidate curve is then updated as to take into account the additional connection with the unambiguous portions. The candidate curve tallying with the minimum cost is then chosen as the optimal ambiguous curve portion. A smooth and continuous representation of the device is then recovered by fitting a approximating cubic B-spline over the retrieved ordered list of oriented 3D segments.

\section{EVALUATION}

We conducted 3 types of experiments to evaluate our method. The first experiment aims at evaluating the ability of our method to reconstruct tortuous 3D curves using a large set of simulated 3D curves. The second experiment was performed using phantom acquisitions of a guide-wire navigation under fluoroscopic guidance in order to evaluate our method against real segmentation results. The third set of results were obtained using fluoroscopic images of a guide-wire navigation performed during a neuro-intervention on a real patient. Implementation of the reconstruction method was done without multi-threading or any specific optimizations on an Intel i7, 2.70 $\mathrm{GHz}$ and 8 GB RAM.

\subsection{Simulated data}

We used cone-beam CT (CBCT) reconstructions of two injected cerebral vascular trees from patients being treated for an arterio-venous malformation (AVM) to simulate a large set of tortuous 3D curves (figure 5). 6 paths to the AVM were identified per patient. Their centerlines were extracted as cubic B-splines. A 3D curve was sampled every $1 \mathrm{~mm}$ along each B-spline curve with a constant length of $2 \mathrm{~cm}$ resulting in a set of 2289 3D curves. Micro guide-wire lengths for neuro-interventions typically range from 2 to $20 \mathrm{~cm}$ but $2 \mathrm{~cm}$ was enough for us to capture already tortuous conformations. Each sampled curve was numerically projected on two image planes using calibrated projection matrices. They were set to model projections on orthogonal frontal and lateral detector planes which is a common configuration for neuro-interventions. Among the 2289 simulated curves, only 1336 curves generating ambiguous epipolar bands were considered for the evaluation (which concerns more than $58 \%$ of the initial set of curves) in order to reflect the effective capacity of our method to reconstruct the correct curve in ambiguous situations. For each curve reconstruction case we assessed the error to the simulated curve from both the set of 3D segments selected as the solution and the B-spline curve fit over those 3D segments. Figure 6 illustrates with an example the curve reconstruction method and shows in red the evaluated output : 3D segments and B-spline fit. We compared different combinations of settings for our reconstruction method. We set our reference setting combination to be (i), where no overlaps were permitted following assumption (1), both distance and orientation were considered equally with $\alpha=0.5$ and all bands spanning more than 3 epipolar lines were processed. In combination (ii) the impact of assumption (2) on the reconstruction error and time performance was studied. In combination (iii), we focused on the cost function to see the impact of considering only the distance on the reconstruction error. Last in (iv) we purposefully ignored bands generating 3D segments that were all shorter than 2.0 voxels to observe the impact on both the error to the ground truth and the gain in running-time performance. 

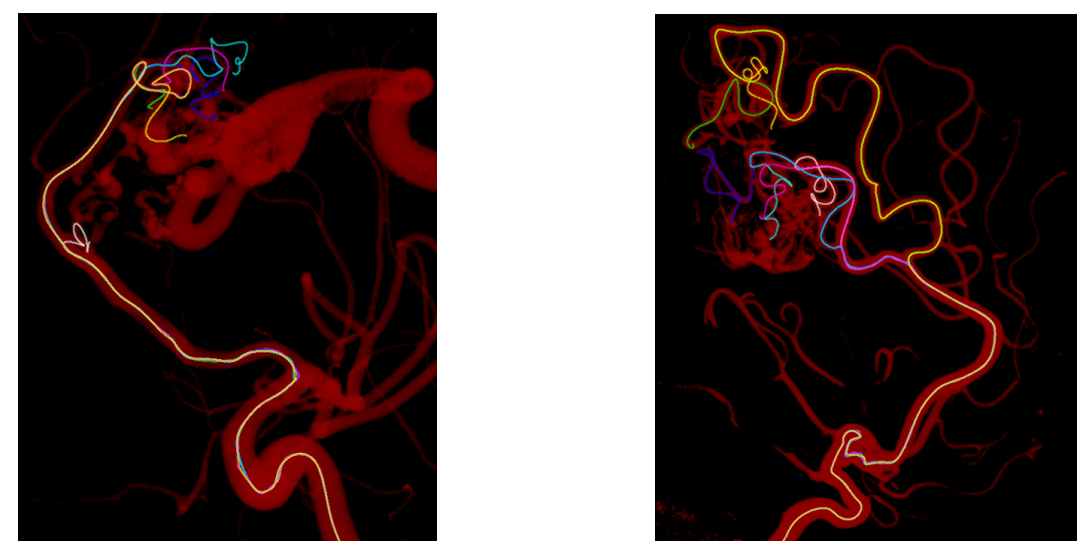

Figure 5: 3D B-spline curves, retrieved from 2 patients' vasculatures, simulating each a different path to an AVM

\subsection{Phantom data}

We used a cerebral vascular phantom (Elastra,Geneva) imaged on an Innova 3131IQ biplane vascular system (GE Healthcare). A micro guide-wire with a slightly curved tip and $2 \mathrm{~cm}$ radio-opaque distal length was inserted in the phantom. X-ray fluoroscopic images were acquired in 5 static positions of the guide-wire. The micro guide-wire was automatically segmented in each projection for the reconstruction. A 3D ground truth was computed for each static position using a CBCT acquisition. The error to the $3 \mathrm{D}$ ground truth was measured as the distance to the centerline of the guide-wire extracted from the CBCT reconstruction.. These include segmentation errors, where more than the guide-wire itself was segmented.

\subsection{Clinical data}

Our data set consists of one sequence of biplane fluoroscopic images depicting the projection of a moving microguide with a radio-opaque distal length of $2 \mathrm{~cm}$. Since no $3 \mathrm{D}$ ground truth was available, we performed a qualitative evaluation based on the re-projection of the B-spline fit performed of the solution set of 3D segments. We selected two configurations where accurate segmentation is difficult to obtain because of the cusp effect and high curvatures. We evaluated the quality of the initial segmentation to a $2 \mathrm{D}$ ground truth of the guide-wire over 63 pairs of projections. In order to assess a robustness to noisy $2 \mathrm{D}$ segments, we confronted this result with the quality of the segmentation when all 2D segments left unmatched after the reconstruction process were removed.

\section{RESULTS}

\subsection{Simulated data}

The four curves of Figure 7 depict the cumulative histogram of errors, all cases included, for the four settings of the algorithm. These errors correspond to the mean distance in $\mathrm{mm}$ of a $3 \mathrm{D}$ segment selected as part of a solution to the ground truth. Figure 7 reports on the vertical axis, the percentage of segments belonging to the solution, that display an error below the number reported on the horizontal axis. The light blue curve corresponds to the worst setting (setting (iii)) where only a distance criteria was considered. It lies just below $95 \%$ for an error of 1 voxel $(0.226 \mathrm{~mm})$. All three other curves are above $97 \%$. For an error of $1.0 \mathrm{~mm}$ marked by the second vertical line, we see that all curves have reached more than $99 \%$ except again for the light blue curve. These results show that it is preferable to include the curvature criteria to perform the reconstruction. Furthermore, the green curve (setting (i)) follows closely the red curve (setting (ii)) showing that using assumption (1) is not too restrictive as compared to its relaxed version assumption (2). Finally we see with the blue curve (setting (iv)) that almost as good results are obtained with the band removal option. The blue and green curves of Figure 8 respectively depict on a logarithmic scale the error in voxels to the simulated data of the reconstructed 3D points using assumption (1) and the B-spline fit of those points. We see that mean error for the B-spline is higher than the one for the 3D segments but remains in average under 1.0 voxel error in more than $94 \%$ of cases. The red curve accounts for the maximum average error committed by the B-spline fit. We see with these results 
that a B-spline fit over the 3D segments accounts well for the unreconstructed portions corresponding to epipolar bands spanning less than 3 epipolar lines. Figure 9 reports time performances of the algorithm for the three settings having an impact on the combinatory. The blue curve (setting (iv)), where assumption (1) is coupled with the band removal option, exhibits the best time performances with a maximum computation time of $13.5 \mathrm{~s}$ in the worst case and a mean computation time of $0.16 \mathrm{~s}$. As a conclusion, setting (iv) provides similar results in accuracy and improved time performance when compared to the reference setting (i). Therefore, choosing to apply assumption (1) and not processing bands generating 3D segments shorter than 2.0 voxels in the curve extraction process seems to be a good compromise for a real-time application.
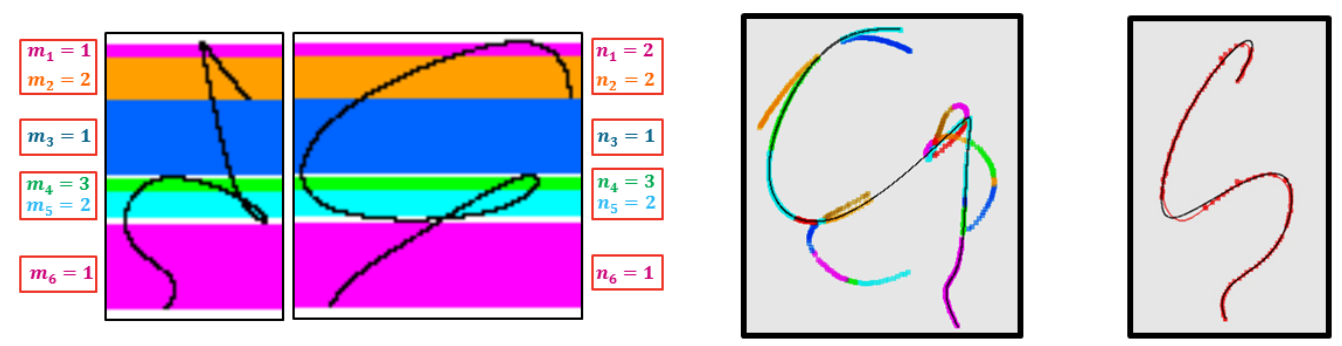

Figure 6: Left : 2 simulated curve projections and with their epipolar bands $\left[m_{b}, n_{b}\right]$. Red boxes depict groups of epipolar bands. Middle : generated 3D segments. Right : simulated curve (black),support (red points), B-spline fit (red).

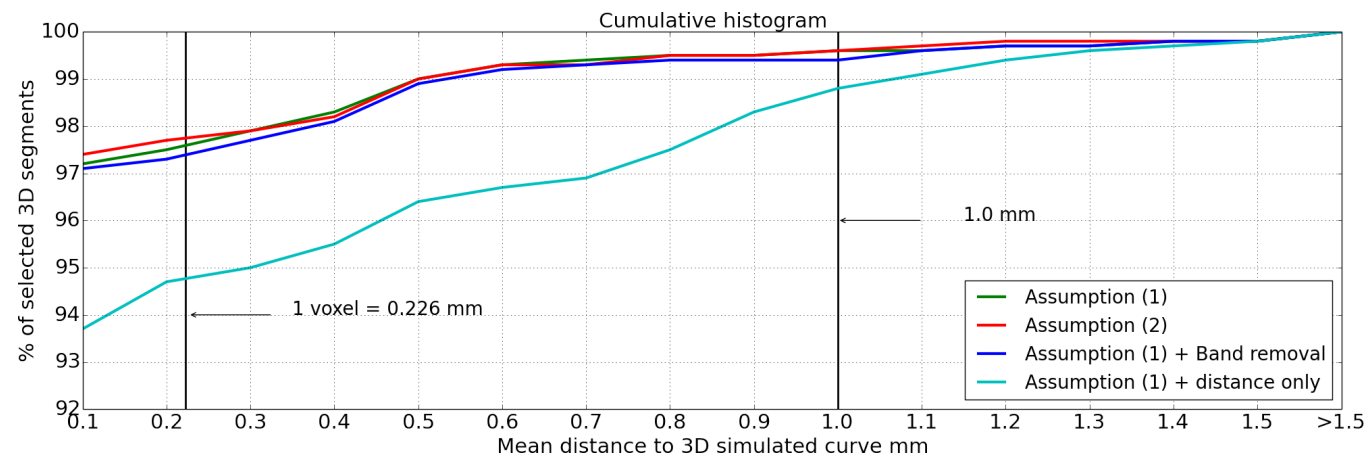

Figure 7: Percentage of 3D segments selected as part of a solution relatively to the mean distance to their corresponding simulated curves. Result correspond to settings (i) green, (ii) red, (iii) cyan and (iv) in blue.

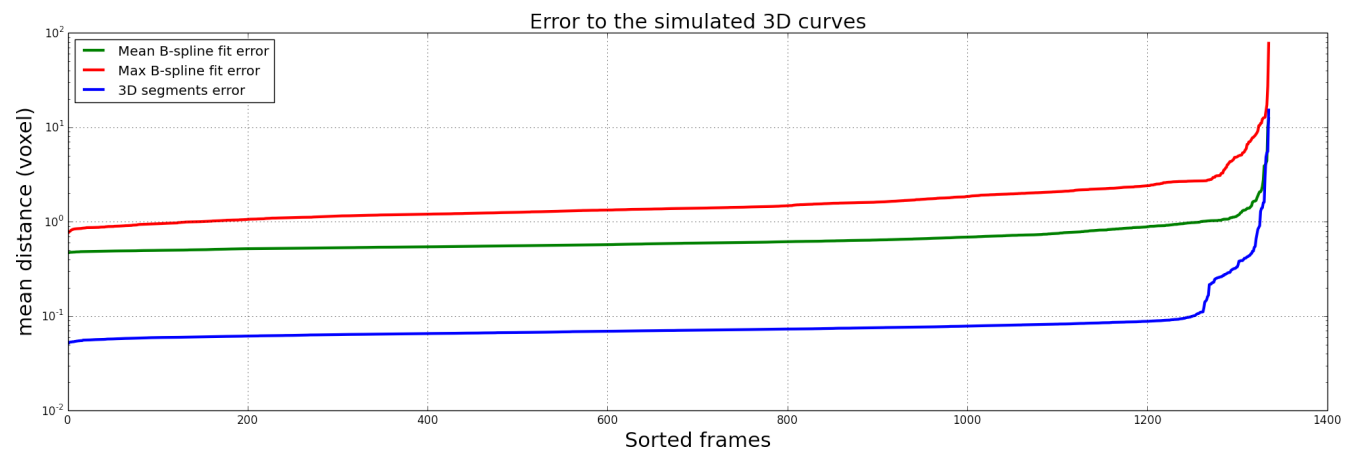

Figure 8: For each case : mean and maximum 3D errors (voxels) for the B-spline fit and mean error for the selected 3D segments sorted in ascending order. Results correspond to the reference setting (i). 


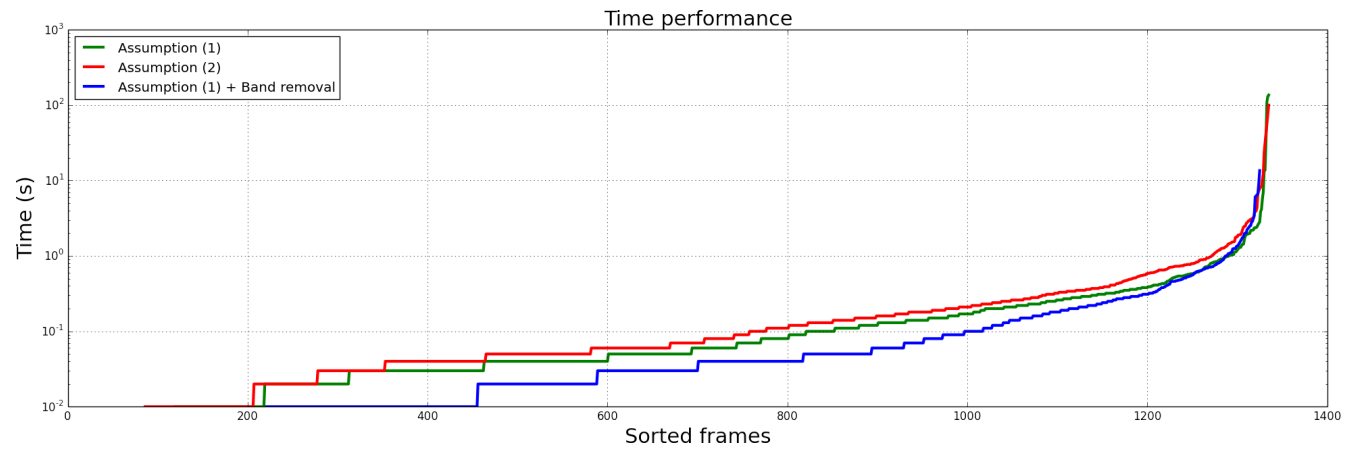

Figure 9: Time performance (seconds) for each case sorted in ascending order. Time results correspond to settings (i) green, (ii) red and (iii) blue.

\subsection{Phantom data}

In Table 1 we report the computed mean distance of the solution fitted by a B-spline to the ground truth for each static position. Over all 5 positions we were able to reconstruct the guide wire accurately with a mean error of $0.46 \mathrm{~mm} \pm 0.16 \mathrm{~mm}$ with a maximum error of $1.46 \mathrm{~mm}$. Table 2 reports the computed mean distance between the ground truth tip positions and the tips of the solution fitted by a B-spline, for each position. This measure reflects how much guide-wire length was missed when reconstructing the $3 \mathrm{D}$ curve. We measured an overall tip error of $0.54 \mathrm{~mm} \pm 0.22 \mathrm{~mm}$ with a maximum error of $6.65 \mathrm{~mm}$.

\begin{tabular}{|c|c|c|c|c|}
\hline \multicolumn{5}{|c|}{ B-spline to ground truth distance (mm) } \\
\hline Position & Nb frames & Mean & Max & Std \\
\hline 1 & 32 & 0.59 & 1.01 & 0.03 \\
\hline 2 & 37 & 0.53 & 1.46 & 0.02 \\
\hline 3 & 30 & 0.52 & 1.32 & 0.04 \\
\hline 4 & 50 & 0.24 & 0.84 & 0.03 \\
\hline 5 & 17 & 0.73 & 0.91 & 0.03 \\
\hline $\mathbf{1 - 5}$ & $\mathbf{1 6 8}$ & $\mathbf{0 . 4 6}$ & $\mathbf{1 . 4 6}$ & $\mathbf{0 . 1 6}$ \\
\hline \multicolumn{5}{|c}{ Table 1: Mean curve error (mm) } \\
\hline
\end{tabular}

\begin{tabular}{|c|c|c|c|c|}
\hline \multicolumn{5}{|c|}{ B-spline tip to ground truth tip distance (mm) } \\
\hline Position & Nb frames & Mean & Max & Std \\
\hline 1 & 32 & 0.59 & 1.09 & 0.29 \\
\hline 2 & 37 & 1.43 & 3.14 & 0.97 \\
\hline 3 & 30 & 2.27 & 6.65 & 1.83 \\
\hline 4 & 50 & 0.95 & 1.66 & 0.28 \\
\hline 5 & 17 & 2.16 & 5.30 & 0.14 \\
\hline $\mathbf{1 - 5}$ & $\mathbf{1 6 8}$ & $\mathbf{1 . 3 4}$ & $\mathbf{6 . 6 5}$ & $\mathbf{1 . 1 9}$ \\
\hline \multicolumn{5}{|c|}{ Table 2: Tip error (mm) } \\
\hline
\end{tabular}

\subsection{Clinical data}

Figure 10 compares the mean distance (in pixels) of the initial segmentation to the ground truth on the horizontal axis to the mean distance of the segmentation without unmatched segments to the ground truth on vertical axis. Blue points mark results for frontal projection cases while the red ones account for the lateral projections. Cases located below the $x=y$ line correspond to cases where the segmentation without the unmatched 2D segments is closer to the ground truth than the initial segmentation. On the contrary, the closer to the $x=y$ line the less significant the changes between the initial segmentation and the one without unmatched 2D segments are relatively to the ground truth. All cases lying in the bottom right quarter of this scatter-graph are cases where the rejection of $2 \mathrm{D}$ segments allowed to bring down the mean error to the ground truth bellow 1.0 pixel error. These preliminary results reflect the robustness of our reconstruction method to the presence of occasional noisy segments in the segmentation. Figures 11 and 12 respectively show a guide-wire forming a cusp and a high curvature in their frontal projection. The re-projection of the solution curve depicted in red for both cases demonstrates how the algorithm was able to recover the correct projected shape by performing a B-spline fit over the solution set of $3 \mathrm{D}$ segments even though the initial segmentation was inaccurate in those locations. 


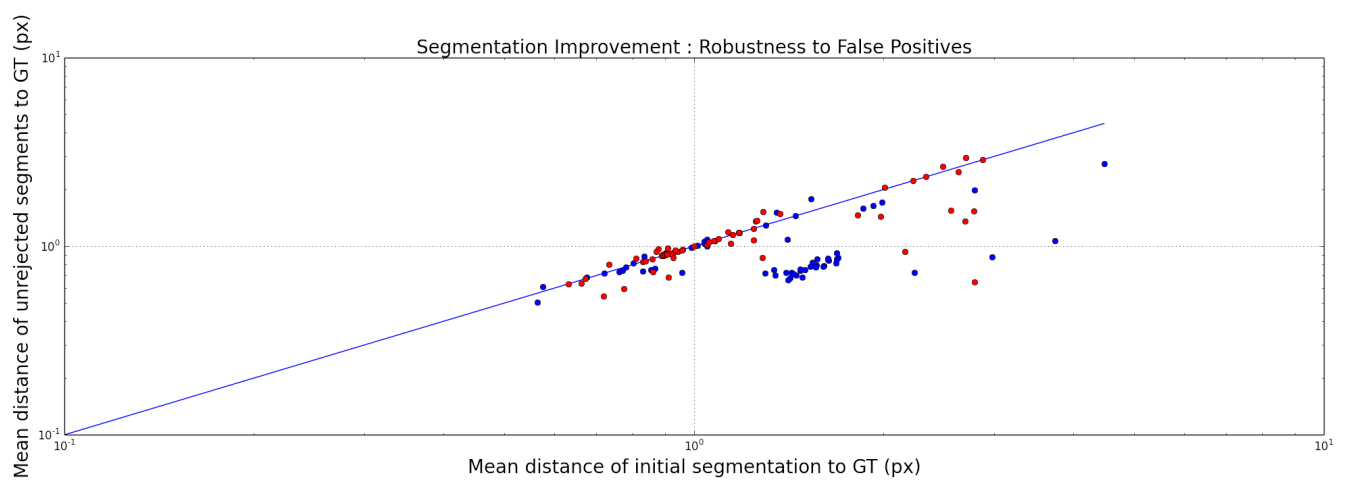

Figure 10: Robustness to noisy 2D segments in both views
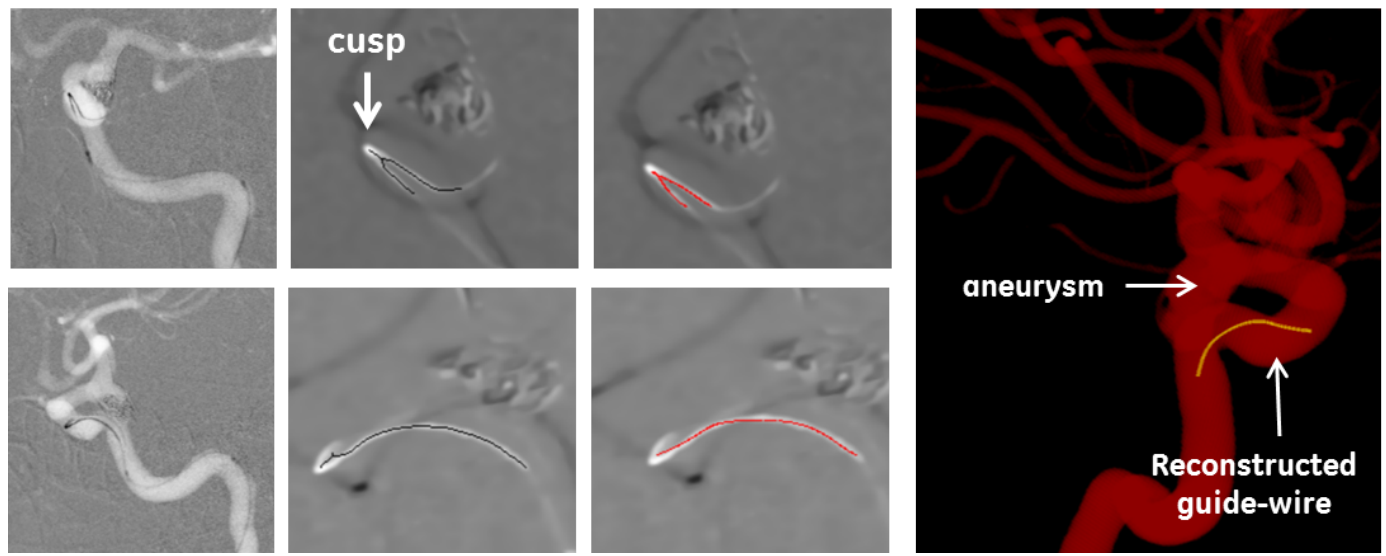

Figure 11: Guide wire reconstructed from 2 views. Top images correspond to the frontal view, bottom images correspond to the lateral view. From left to right : Fluoroscopic image, result of segmentation performed on a filtered and rectified image, forward-projection of the 3D spline fit of the solution 3D curve segments. The right-hand most image depicts the reconstructed guide wire registered with the corresponding vascular tree.
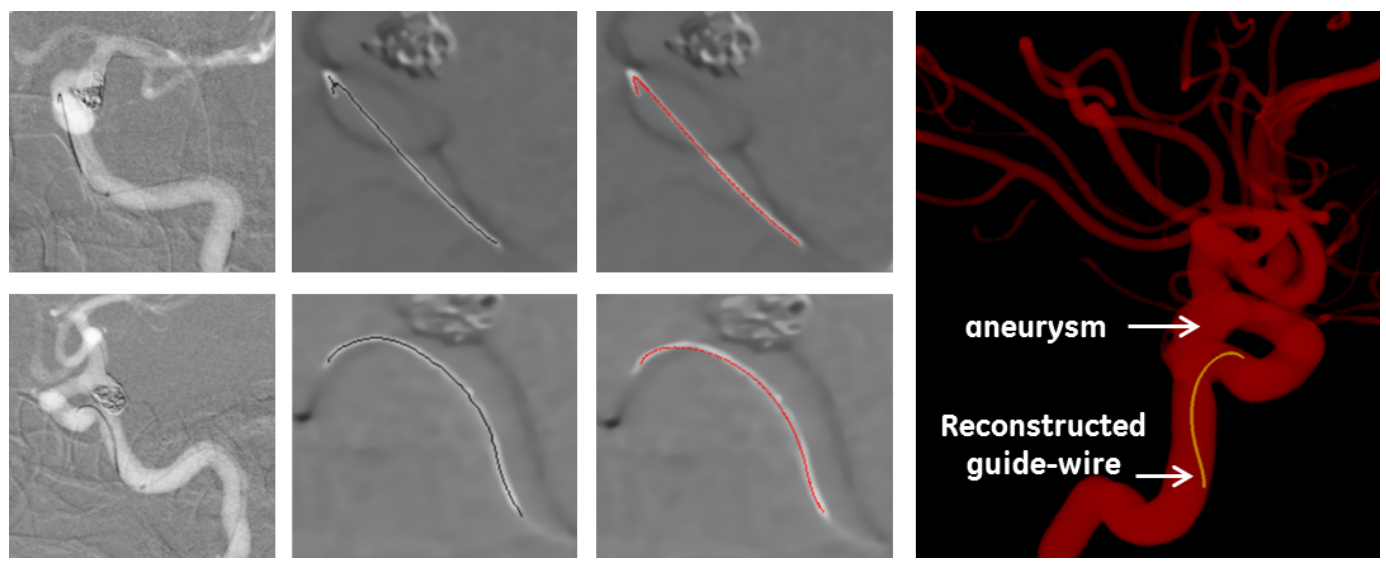

Figure 12: Guide wire reconstructed from 2 views with the lateral view presenting a high curvature at the tip not accurately segmented. Top images correspond to the frontal view, bottom images correspond to the lateral view. From left to right : Fluoroscopic image, result of segmentation performed on a filtered and rectified image, forward-projection of the 3D spline fit of the solution 3D curve segments. The right-hand most image depicts the reconstructed guide wire registered with the corresponding vascular tree. 


\section{CONCLUSION}

We presented a new 3D curve reconstruction method from two views able to handle tortuous $3 \mathrm{D}$ curves. The method relies on the generation of 3D curve segments from which a subset minimizing a function of both length and curvature can be extracted to form the 3D curve. It is achieved using a graph representation as to extract sets of 3D curve segments satisfying a matching constraint that minimizes the number of overlaps in the projections and orders them independently using 3D geometric constraints. The ordered set of 3D curve segments that best minimizes those constraints is then chosen as the optimal 3D curve solution. On the one hand, experiments show that ambiguous situations arise frequently in the cerebral vascular tree (especially in a context of AVM) and that our algorithm is able to handle those ambiguities with an ideal segmentation input. On the other hand, preliminary experiments on phantom and clinical data show that our algorithm still behaves well when confronted with over segmented data since we can rely on unambiguous segments that will necessarily be part of the final solution. Future works will further address the management of imperfect segmented input data particularly when gaps and over segmentation occur both within the same epipolar band.

\section{REFERENCES}

[1] Baert, S. A. M., van de Kraats, E., van Walsum, T., Viergever, M., and Niessen, W., "Three-dimensional guide-wire reconstruction from biplane image sequences for integrated display in 3-d vasculature," Medical Imaging, IEEE Transactions on 22, 1252-1258 (Oct 2003).

[2] Hoffmann, M., Brost, A., Jakob, C., Bourier, F., Koch, M., Kurzidim, K., Hornegger, J., and Strobel, N., "Semi-Automatic Catheter Reconstruction from Two Views," in [MICCAI 2012, Part I], Ayache, N., Delingette, H., Golland, P., and Mori, K., eds., Lecture Notes in Computer Science, 584-591 (2012).

[3] Petkovic, T., Loncaric, S., Devcic, T., and Homan, R., "Non-iterative guidewire reconstruction from multiple projective views," in [Image and Signal Processing and Analysis (ISPA), 2011 7th International Symposium on], 639-643 (Sept 2011).

[4] Spiegel, M., Pfister, M., Hahn, D., Daum, V., Hornegger, J., Struffert, T., and Drfler, A., "Towards real-time guidewire detection and tracking in the field of neuroradiology," Proc. SPIE 7261, 726105-726105-8 (2009).

[5] Lessard, S., Lau, C., Chav, R., Soulez, G., Roy, D., and de Guise, J. A., "Guidewire tracking during endovascular neurosurgery," Medical Engineering \& Physics 32(8), 813 - 821 (2010).

[6] Bismuth, V., Vaillant, R., Talbot, H., and Najman, L., "Curvilinear structure enhancement with the polygonal path image - application to guide-wire segmentation in x-ray fluoroscopy," in [Medical Image Computing and Computer-Assisted Intervention MICCAI 2012], Ayache, N., Delingette, H., Golland, P., and Mori, K., eds., Lecture Notes in Computer Science 7511, 9-16, Springer Berlin Heidelberg (2012).

[7] Li, G. and Zucker, S., "Contextual inference in contour-based stereo correspondence," International Journal of Computer Vision 69(1), 59-75 (2006).

[8] Kahl, F. and August, J., "Multiview reconstruction of space curves," in [Computer Vision, 2003. Proceedings. Ninth IEEE International Conference on], 1017-1024 vol.2 (Oct 2003).

[9] Hartley, R. and Zisserman, A., [Multiple View Geometry in Computer Vision], Cambridge University Press, New York, NY, USA, 2 ed. (2003). 